\title{
The role of the $N^{*}(2080)$ resonance in the $\vec{\gamma} p \rightarrow K^{+} \Lambda(1520)$ reaction
}

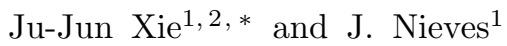 \\ ${ }^{1}$ Instituto de Física Corpuscular (IFIC), Centro Mixto CSIC-Universidad de Valencia, \\ Institutos de Investigación de Paterna, Aptd. 22085, E-46071 Valencia, Spain \\ ${ }^{2}$ Department of Physics, Zhengzhou University, Zhengzhou, Henan 450001, China
}

(Dated: November 10, 2018)

\begin{abstract}
We investigate the $\Lambda(1520)$ photo-production in the $\vec{\gamma} p \rightarrow K^{+} \Lambda(1520)$ reaction within the effective Lagrangian method near threshold. In addition to the "background" contributions from the contact, $t$-channel $K$ exchange, and $s$-channel nucleon pole terms, which were already considered in previous works, the contribution from the nucleon resonance $N^{*}(2080)$ (spin-parity $J^{P}=3 / 2^{-}$) is also considered. We show that the inclusion of the nucleon resonance $N^{*}(2080)$ leads to a fairly good description of the new LEPS differential cross section data, and that these measurements can be used to determine some of the properties of this latter resonance. However, serious discrepancies appear when the predictions of the model are compared to the photon-beam asymmetry also measured by the LEPS Collaboration.
\end{abstract}

PACS numbers: 13.75.Cs.; 14.20.-c.; 13.60.Rj.

\section{INTRODUCTION}

The $\Lambda(1520)\left(\equiv \Lambda^{*}\right)$ photo-production in the $\gamma p \rightarrow$ $K^{+} \Lambda^{*}$ reaction is an interesting tool to gain a deeper understanding of the interaction among strange hadrons and also on the nature of baryon resonances. There have been some experimental efforts dedicated to this reaction. There exist measurements from two old experiments in the high energy region $E_{\gamma}=2.8-4.8 \mathrm{GeV}$ by the LAMP2 Collaboration [1], and $E_{\gamma}=11 \mathrm{GeV}$ by Boyarski et al. 2]. Recently, this reaction has been examined at photon energies below $2.4 \mathrm{GeV}$ in the SPring-8 LEPS experiment [3, 4]. For an invariant $\gamma p$ mass $W \simeq 2.11$ $\mathrm{GeV}$, this latter experiment has reported a new bump structure in the differential cross section at forward $K^{+}$ angles, which might hint to a sizeable contribution from nucleon resonances in the $s$-channel.

On the theoretical side, there exist several effective hadron Lagrangian studies of this reaction for laboratory photon energies ranging from threshold $(\approx 1.7 \mathrm{GeV})$, up to about $5 \mathrm{GeV}$, where the old experimental measurements were available. Different dominant mechanisms have been proposed to describe the LAMP2 high energy results for angular and energy differential cross sections. Thus, in Refs. [5, 6] it is claimed a large contribution from the $t$-channel $K^{*}$ exchange, while a large contribution from a gauge invariant contact term along with $t$-channel $K$ exchange and baryon pole $(s-$ and $u-$ channels) is advocated in [7, 8]. On the other hand, a quark-gluon string reaction mechanism, which was realized in the $K$ meson Reggeon exchange model, is also able to reproduce the available experimental data in the high photon energy region from $E_{\gamma} \simeq 2.8 \mathrm{GeV}$ up to $5 \mathrm{GeV}$, as discussed in [9]. In this latter work, the coupling of the $\Lambda^{*}$ resonance, which is dynamically generated within

*Electronic address: xiejujun@ific.uv.es a chiral unitary model [10], to the $N \bar{K}^{*}$ pair is shown to be quite small and hence, the contribution of $t$-channel $K^{*}$ exchange is taken to be much smaller than that due to exchange of the pseudoscalar $K$ meson. Indeed, this minor role played by the $K^{*}$ exchange gets support from the decay asymmetry measured by the CLAS Collaboration [11]. Besides, the photon beam asymmetry for $K \Lambda^{*}$ photo-production has been also proposed in Ref. [12] as tool to unravel the reaction mechanism, since it is predicted to be very small for the contact and $s-$ and $u-$ baryon pole terms, while much larger for $t$-channel $K$ and $K^{*}$ exchanges. Moreover, $K$ and $K^{*}$ exchanges give rise to different signs of the photon-beam asymmetry [12].

All these models fail, however, to describe the forward bump structure that appears in the new LEPS differential cross section data at low energies.

In the present work, we reanalyze the $\vec{\gamma} p \rightarrow K^{+} \Lambda^{*}$ reaction within the effective Lagrangian method nearthreshold, where the new experimental data from the LEPS Collaboration [4] have been taken. In addition to the "background" contributions from the contact, $t$-channel $K$ exchange, and $s$-channel nucleon pole terms, which were already considered in previous works, we have also studied possible contributions from nucleon resonances aiming at describing the bump at forward angles reported by LEPS. Unfortunately, the information about nucleon resonances in the relevant mass region $(\sim 2.1) \mathrm{GeV}$ is scarce [13], which means that the evidence of their existence and the knowledge of their properties are poor. Thus, it is necessary to rely on theoretical schemes, such that of Ref. [14, 15] based in a quark model (QM) for baryons. Among the possible nucleon resonances, we have finally considered only the two-star $D$-wave $J^{P}=3 / 2^{-} N^{*}(2080)\left(\equiv N^{*}\right)$ one, which is predicted to have visible contributions 15 to the $\gamma p \rightarrow K^{+} \Lambda^{*}$ reaction. Although the $N^{*}(2080)$ resonance is listed in the Particle Data Group (PDG) book, the evidence of its existence is poor or only fair and further work is required to verify their existence and to know its 
properties, accordingly, its total decay width and branching ratios are not experimentally known, either. In this respect, we show in this work how the LEPS measurements could be used to determine some of the properties of this resonance.

To end this introduction, we would like to mention that in Refs. [8, 16], the role played by the $N^{*}(2080)$ resonance in the $\vec{\gamma} p \rightarrow K^{+} \Lambda^{*}$ reaction has been also studied. In these works, in sharp contrast with our findings, it is pointed out that the $N^{*}(2080)$ resonance has a negligible contribution and therefore its contribution is not expected to explain the bump structure at forward angles reported by LEPS. This is greatly due to the small $N^{*}(2080) \Lambda^{*} K^{+}$coupling and large width of the resonance used in Refs. [8, 16], and we will comment on this below.

The paper is organized as follows. In Sect. III we shall discuss the formalism and the main ingredients of the model, while our results and conclusions are presented in Sects. III and [V]

\section{FORMALISM AND INGREDIENTS}

The basic tree level Feynman diagrams for the $\vec{\gamma} p \rightarrow$ $K^{+} \Lambda^{*}$ reaction are depicted in the Fig. 1. These include the $t$-channel $K$ exchange, $s$-channel nucleon and nucleon resonance, and contact terms. To compute the contributions of these terms, we use the interaction Lagrangian densities of Refs. [8, 9],

$$
\begin{aligned}
\mathcal{L}_{\gamma K K}= & -i e\left(K^{-} \partial^{\mu} K^{+}-K^{+} \partial^{\mu} K^{-}\right) A_{\mu} \\
\mathcal{L}_{K p \Lambda^{*}}= & \frac{g_{K N \Lambda^{*}} \bar{\Lambda}^{* \mu}\left(\partial_{\mu} K^{-}\right) \gamma_{5} p+\text { h.c. }}{m_{K}}= \\
\mathcal{L}_{\gamma p p}= & -e \bar{p}\left(A-\frac{\kappa_{p}}{2 M_{N}} \sigma_{\mu \nu}\left(\partial^{\nu} A^{\mu}\right)\right) p+\text { h.c. } \\
\mathcal{L}_{\gamma K p \Lambda^{*}}= & -i e \frac{g_{K N \Lambda^{*}}}{m_{K}} \bar{\Lambda}^{* \mu} A_{\mu} K^{-} \gamma_{5} p+\text { h.c. } \\
\mathcal{L}_{\gamma N N^{*}}= & \frac{i e f_{1}}{2 m_{N}} \bar{N}_{\mu}^{*} \gamma_{\nu} F^{\mu \nu} N- \\
& \frac{e f_{2}}{\left(2 m_{N}\right)^{2}} \bar{N}_{\mu}^{*} F^{\mu \nu} \partial_{\nu} N+\text { h.c. } \\
\mathcal{L}_{K \Lambda^{*} N^{*}}= & \frac{g_{1}}{m_{K}} \bar{\Lambda}_{\mu}^{*} \gamma_{5} \gamma_{\alpha}\left(\partial^{\alpha} K\right) N^{* \mu}+ \\
& \frac{i g_{2}}{m_{K}^{2}} \bar{\Lambda}_{\mu}^{*} \gamma_{5}\left(\partial^{\mu} \partial_{\nu} K\right) N^{* \nu}+\text { h.c. }
\end{aligned}
$$

where $e=\sqrt{4 \pi \alpha}>0(\alpha=1 / 137.036$ is the fine-structure constant), $\kappa_{p}=1.79, A_{\mu}$ and $F_{\mu \nu}=\partial_{\mu} A_{\nu}-\partial_{\nu} A_{\mu}$ are the proton charge and magnetic moment, and the photon field and electromagnetic field tensor, respectively. We use the Rarita-Schwinger formalism [17, 18] to describe the spin $J=3 / 2 \Lambda^{*}$ and $N^{*}$ resonances, while the $N^{*}(2080)$ electromagnetic $f_{1,2}$ and hadronic $g_{1,2}$ couplings will be discussed below.

With the effective interaction Lagrangian densities given above, we can easily construct the invariant scat-
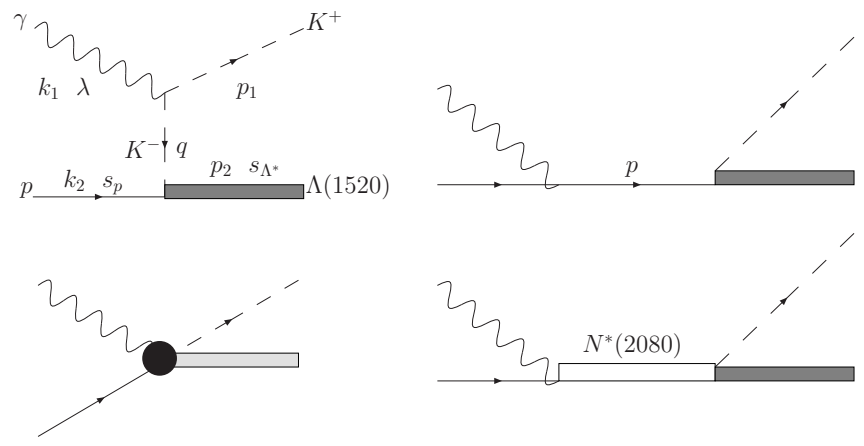

FIG. 1: Model for the $\gamma p \rightarrow \Lambda^{*} K^{+}$reaction. It consists of $t$-channel $K$ exchange, $s$-channel nucleon and nucleon resonance, and contact terms. In the first diagram, we also show the definition of the kinematical $\left(k_{1}, k_{2}, p_{1}, p_{2}\right)$ and polarization variables $\left(\lambda, s_{p}, s_{\Lambda^{*}}\right)$ that we used in the present calculation. In addition, we use $q=k_{1}-p_{1}$

tering amplitudes,

$$
-i T_{i}=\bar{u}_{\mu}\left(p_{2}, s_{\Lambda^{*}}\right) A_{i}^{\mu \nu} u\left(k_{2}, s_{p}\right) \epsilon_{\nu}\left(k_{1}, \lambda\right)
$$

where $u_{\mu}$ and $u$ are dimensionless Rarita-Schwinger and Dirac spinors, respectively, while $\epsilon_{\nu}\left(k_{1}, \lambda\right)$ is the photon polarization vector. The reduced $A_{i}^{\mu \nu}$ amplitudes read.

$$
\begin{aligned}
A_{t}^{\mu \nu}= & -e \frac{g_{K N \Lambda^{*}}}{m_{K}} \frac{1}{q^{2}-m_{K}^{2}} q^{\mu}\left(q^{\nu}-p_{1}^{\nu}\right) \gamma_{5} f_{\mathrm{c}} \\
A_{s}^{\mu \nu}= & -e \frac{g_{K N \Lambda^{*}}}{m_{K}} \frac{1}{s-M_{N}^{2}} p_{1}^{\mu} \gamma_{5}\left\{\not \iota_{1} \gamma^{\nu} f_{\mathrm{s}}+\left(\not k_{2}+M_{N}\right) \gamma^{\nu} f_{\mathrm{c}}\right. \\
& \left.+\left(\not k_{1}+\not k_{2}+M_{N}\right) \mathrm{i} \frac{\kappa_{p}}{2 M_{N}} \sigma_{\nu \rho} k_{1}^{\rho} f_{\mathrm{s}}\right\} \\
A_{c}^{\mu \nu}= & e \frac{g_{K N \Lambda^{*}}}{m_{K}} g^{\mu \nu} \gamma_{5} f_{\mathrm{c}} \\
A_{R}^{\mu \nu}= & \gamma_{5}\left(\frac{g_{1}}{m_{K}} p_{1} g^{\mu \rho}-\frac{g_{2}}{m_{K}^{2}} p_{1}^{\mu} p_{1}^{\rho}\right) \frac{\not k_{1}+\not k_{2}+M_{N^{*}}}{s-M_{N^{*}}^{2}+i M_{N^{*}} \Gamma_{N^{*}}} \\
& P_{\rho \sigma}\left(\frac{e f_{1}}{2 m_{N}}\left(k_{1}^{\sigma} \gamma^{\nu}-g^{\sigma \nu} \not k_{1}\right)+\frac{e f_{2}}{\left(2 m_{N}\right)^{2}}\right. \\
& \left.\left(k_{1}^{\sigma} k_{2}^{\nu}-g^{\sigma \nu} k_{1} \cdot k_{2}\right)\right) f_{R}
\end{aligned}
$$

The sub-indices $t, s, c$ and $R$ stand for the $t$-channel kaon exchange, $s$-channel nucleon pole, contact, and resonance $N^{*}$ pole terms ${ }^{1}$, and

$$
\begin{aligned}
P_{\rho \sigma}= & -g_{\rho \sigma}+\frac{1}{3} \gamma_{\rho} \gamma_{\sigma}+\frac{2}{3 M_{N^{*}}^{2}}\left(k_{1}+k_{2}\right)_{\rho}\left(k_{1}+k_{2}\right)_{\sigma} \\
& +\frac{1}{3 M_{N^{*}}}\left(\gamma_{\rho}\left(k_{1}+k_{2}\right)_{\sigma}-\gamma_{\sigma}\left(k_{1}+k_{2}\right)_{\rho}\right) .
\end{aligned}
$$

\footnotetext{
${ }^{1}$ We do not consider in this work the $t$-channel $K^{*}$ exchange and the $u$-channel hyperon pole terms, since we expect these contributions to be small, as it was discussed in Ref. [9].
} 
Note, that there exist some ambiguities when dealing with the propagation and couplings of spin $3 / 2$ off-shell particles [19 21]. In this exploratory work, we ignore this problem, since we are using a tree level approach and possible effects might be partially effectively encoded into the phenomenological $N^{*}$ hadron couplings, which will be fitted to data. This interesting issue deserves future detailed research. Besides, $M_{N^{*}}$ and $\Gamma_{N^{*}}$ are the mass and the total decay width of the $N^{*}$ resonance. We have examined here two different scenarious. In the first one, we set $M_{N^{*}}=2.08 \mathrm{GeV}$, as quoted in the PDG [13]. On the other hand, since $\Gamma_{N^{*}}$ has a large experimental uncertainty [13], in this first scenarious, we use $\Gamma_{N^{*}}=300$ $\mathrm{MeV}$ for the numerical calculations. These values have been also used in Ref. [22], where the contributions of the $N^{*}$ resonance in the $\gamma p \rightarrow K \Sigma(1385)$ reaction were examined, and found to give important contributions to this reaction. In the second scenarious, we have fitted both, the mass and the total width of the $N^{*}$ resonance to the LEPS differential cross section data.

Up to this point, the $T$-matrix is gauge invariant. However, we ought to introduce the compositeness of the hadrons. This is usually achieved by including formfactors in the amplitudes in such manner that gauge invariance is preserved ${ }^{2}$. There is no an unique theoretical way to introduce the form-factors, this was discussed at length in the late nineties $[23-26]$. We adopt here the scheme used in the previous works [5, 7, 8], where the prescription of Ref. [24] was used. We take the following parameterization for the four-dimensional form-factors

$$
\begin{aligned}
& f_{\mathrm{i}}=\frac{\Lambda_{i}^{4}}{\Lambda_{i}^{4}+\left(q_{\mathrm{i}}^{2}-M_{\mathrm{i}}^{2}\right)^{2}}, \quad \mathrm{i}=\mathrm{s}, \mathrm{t}, \mathrm{R} \\
& f_{\mathrm{c}}=f_{\mathrm{s}}+f_{\mathrm{t}}-f_{\mathrm{s}} f_{\mathrm{t}}, \quad \text { and }\left\{\begin{array}{l}
q_{\mathrm{s}}^{2}=q_{\mathrm{R}}^{2}=s, q_{\mathrm{t}}^{2}=q^{2} \\
M_{\mathrm{s}}=M_{N} \\
M_{\mathrm{R}}=M_{N^{*}} \\
M_{\mathrm{t}}=m_{K}
\end{array}\right.
\end{aligned}
$$

We will consider different cut-off values for the background and resonant terms, i.e. $\Lambda_{s}=\Lambda_{t} \neq \Lambda_{R}$.

In the expressions of the different contributions to the $T$ amplitude, given in Eqs. (8)-(11), we have already included the form-factors. The form of $f_{\mathrm{c}}$ is chosen such that the on-shell values of the coupling constants are reproduced. For the sake of simplicity, we neglect the terms affected by the $f_{s}$ form-factor ${ }^{3}$, since they are greatly suppressed by it [7, 8].

We take $g_{K N \Lambda^{*}}=10.5$, as determined from the $\Lambda^{*} \rightarrow p K^{-}$decay width (we use for the full decay width $\Gamma_{\Lambda^{*}}=15.6 \mathrm{MeV}$ and a value of 0.45 for the $\Lambda^{*} \rightarrow \bar{K} N$ branching ratio [13]), while the $N^{*} N \gamma$ coupling constants

\footnotetext{
${ }^{2}$ For the sake of brevity and to avoid repeating similar equations in Eqs. (8)-11] we have already included form-factors $\left(f_{s}, f_{c}, f_{R}\right)$. Details are given in what follows.

3 Those terms are gauge-invariant by themselves.
}

$f_{1}$ and $f_{2}$ could be fixed, in principle, from the $N^{*}$ helicity amplitudes $A_{1 / 2}$ and $A_{3 / 2}$ [22],

$$
\begin{aligned}
A_{1 / 2}^{p^{*}}= & \frac{e \sqrt{6}}{12} \sqrt{\frac{k_{\gamma}}{M_{N} M_{N^{*}}}} \\
& \left(f_{1}+\frac{f_{2}}{4 M_{N}^{2}} M_{N^{*}}\left(M_{N^{*}}+M_{N}\right)\right), \\
A_{3 / 2}^{p^{*}}= & \frac{e \sqrt{2}}{4 M_{N}} \sqrt{\frac{k_{\gamma} M_{N^{*}}}{M_{N}}} \\
& \left(f_{1}+\frac{f_{2}}{4 M_{N}}\left(M_{N^{*}}+M_{N}\right)\right)
\end{aligned}
$$

where $k_{\gamma}=\left(M_{N^{*}}^{2}-M_{N}^{2}\right) /\left(2 M_{N^{*}}\right)$, and the the superscript $p^{*}$ indicates the positive-charge $D_{13}$ resonance. The values of $f_{1}$ and $f_{2}$ (in units of the proton charge $e$ ) deduced from the helicity amplitudes quoted in the PDG [13] are listed in the Table I] Finally, the strong couplings $g_{1}, g_{2}$ and the cut-off parameters $\Lambda_{s}=\Lambda_{t}$ and $\Lambda_{R}$, appearing in the form factors, are free parameters in the present calculation, and we will fit them to the $\gamma p \rightarrow K^{+} \Lambda$ (1520) differential cross section data below $E_{\gamma}=2.4 \mathrm{GeV}$ reported in Ref. [4].

\section{NUMERICAL RESULTS AND DISCUSSION}

The differential cross section, in the center of mass frame (C.M.), and for a polarized photon beam reads,

$$
\begin{aligned}
\left.\frac{d \sigma}{d \Omega}\right|_{\text {C.M. }} & =\frac{\left|\vec{k}_{1}^{\text {C.M. }}\right|\left|\vec{p}_{1}^{\text {C.M. }}\right|}{4 \pi^{2}} \frac{M_{N} M_{\Lambda^{*}}}{\left(s-M_{N}^{2}\right)^{2}}\left(\frac{1}{2} \sum_{s_{p}, s_{\Lambda}^{*}}|T|^{2}\right) \\
= & \frac{1}{2 \pi} \frac{d \sigma}{d\left(\cos \theta_{\text {C.M. }}\right)}\left\{1-\Sigma \cos 2\left(\phi_{\text {C.M. }}-\alpha\right)\right\}(17)
\end{aligned}
$$

where we have taken the photon momentum in the positive $Z$-axis direction, $\theta_{\text {C.M. }}$. and $\phi_{\text {C.M. }}$. are the polar and azimuthal outgoing $K^{+}$scattering angles, $\vec{k}_{1}$ C.M. and $\vec{p}_{1}^{\text {C.M. }}$ are the photon and $K^{+}$meson c.m. threemomenta, and the photon polarization vector reads $\epsilon^{\mu}=$ $(0, \cos \alpha, \sin \alpha, 0)$ [see Fig. 2]. The asymmetry function ${ }^{4}$ $\Sigma$ depends on $\theta_{\text {C.M. }}$, but it does not depend on the azimuthal angle $\phi_{\mathrm{C} . \mathrm{M}}$.

We perform four $\left(g_{1}, g_{2}, \Lambda_{s}=\Lambda_{t}\right.$ and $\left.\Lambda_{R}\right)$, six $\left(g_{1}, g_{2}\right.$, $\Lambda_{s}=\Lambda_{t}, \Lambda_{R}, M_{N^{*}}$ and $\left.\Gamma_{N^{*}}\right)$ and eight parameter $\left(e f_{1}\right.$, $e f_{2}, g_{1}, g_{2}, \Lambda_{s}=\Lambda_{t}, \Lambda_{R}, M_{N^{*}}$ and $\left.\Gamma_{N^{*}}\right) \chi^{2}$-fits to the LEPS $d \sigma / d\left(\cos \theta_{\text {C.M. }}\right)$ data at forward angles displayed in the left panels of Fig. 2 of Ref. [4]. There is a total

\footnotetext{
${ }^{4}$ Our definition of this function is consistent with that used in previous theoretical [8, 12] and experimental [3, 4] papers. Thus, for instance and to make contact with the definitions used in Ref. [12], we would have there $\phi=0$, since in this reference the reaction plane is chosen to be the $X Z$ one, while the parallel and perpendicular directions of the photon polarization vector would correspond to $\alpha=0$ and $\alpha=\pi / 2$, respectively.
} 


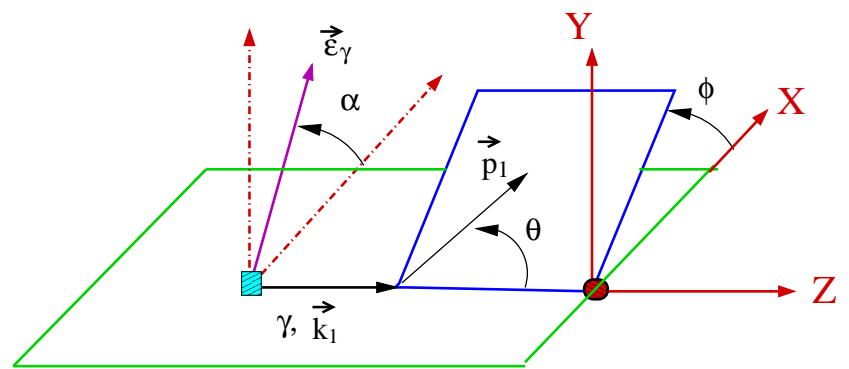

FIG. 2: (Color online) Definition of the different angles used through this work.

of 59 data points. These data correspond to forward $K^{+}$ angles and are given for four intervals of $\cos \theta_{\text {C.M. }}$ ranging from 1 down to 0.6 . To compute the cross sections in each interval we always use the corresponding mean value of $\cos \theta_{\text {C.M. }}$.

The fitted parameters are compiled in Table [ For the first two fits (A and B), in which the couplings $e f_{1}$ and $e f_{2}$ are fixed from the $N^{*}$ helicity amplitudes $A_{1 / 2}$ and $A_{3 / 2}$, we quote two sets of errors for the fitted parameters. The first set of errors is purely statistical and it is determined from the the inverse of the $\chi^{2}$-Hessian matrix at the minimum, while the second set accounts for some systematics of the present approach and it takes into account the errors of $f_{1}$ and $f_{2}$ induced by the PDG helicity amplitude uncertainties. We use a Monte Carlo
(MC) simulation to estimate these latter errors. We generate pairs of couplings $\left(f_{1}, f_{2}\right)$ from a two-dimensional uncorrelated Gaussian distribution with the mean values and standard deviations quoted in Table [1 For each $\left(f_{1}, f_{2}\right)$ pair, we perform a $\chi^{2}$-fit and thus a four dimensional distribution of fitted parameters $\left(g_{1}, g_{2}, \Lambda_{s}=\Lambda_{t}\right.$ and $\left.\Lambda_{R}\right)$ is generated. The second set of errors, given in the Table II. accounts for the corresponding $68 \%$ confidence level (CL) interval deduced from that distribution. Because its completely different origin, one can add in quadratures the two sets of errors quoted in the Table I for fits $\mathrm{A}$ and $\mathrm{B}$. In the case of the fit $\mathrm{C}$ (eight parameters), we just give statistical errors as determined from the the inverse of the $\chi^{2}$-Hessian matrix at the minimum.

We find all fits displayed in the Table【show reasonable small $\chi^{2} /$ dof. We have also performed a best fit including only non-resonant background contributions. It only has one free parameter $\Lambda_{s}=\Lambda_{t}$, which turns out to be $592 \pm 1 \mathrm{MeV}$, and an unacceptable $\chi^{2} / d o f \sim 24$. Thus, the inclusion of the nucleon resonance $N^{*}(2080)$ is crucial to achieve a fairly good description of the new LEPS differential cross section data.

With the strong coupling constants obtained from the $\chi^{2}$-fits, we have evaluated the $N^{*}(2080)$ to $\Lambda^{*} K$ partial decay width,

$$
\begin{aligned}
\Gamma_{N^{*} \rightarrow \Lambda^{*} K} & =\frac{\left|\vec{p}_{1}^{\text {C.M. }}\right| M_{N^{*}}\left(E_{\Lambda^{*}}-M_{\Lambda^{*}}\right)}{18 \pi M_{\Lambda^{*}}^{2}}\left\{\left|\vec{p}_{1}^{\text {C.M. }}\right|^{4} \frac{g_{2}^{2}}{m_{K}^{4}}+\left|\vec{p}_{1}^{\text {C.M. }}\right|^{2}\left(2 E_{\Lambda^{*}}-M_{\Lambda^{*}}\right) \frac{\left(M_{N^{*}}+M_{\Lambda^{*}}\right)}{M_{N^{*}}} \frac{g_{1} g_{2}}{m_{K}^{3}}\right. \\
& \left.+\left(\frac{M_{N^{*}}+M_{\Lambda^{*}}}{M_{N^{*}}}\right)^{2}\left(E_{\Lambda^{*}}^{2}-E_{\Lambda^{*}} M_{\Lambda^{*}}+\frac{5}{2} M_{\Lambda^{*}}^{2}\right) \frac{g_{1}^{2}}{m_{K}^{2}}\right\}
\end{aligned}
$$

as deduced from the Lagrangian of Eq. (6). In the above expression $E_{\Lambda^{*}}=\sqrt{M_{\Lambda^{*}}^{2}+\left|\vec{p}_{1}^{\text {C.M. }}\right|^{2}}$. The numerical predictions $^{5}$ for each fit are also given in the Table I. Let us first pay attention to fit A results. Having in mind that in that case, we have assumed a total width of 300 $\mathrm{MeV}$ for the $N^{*}(2080)$ resonance, we find that the $\Lambda^{*} K$ decay mode of this resonance will be become the dominant one, if one attributes the observed bump structure at forward angles, reported in the SPring-8 LEPS experiment, to the effects produced by this resonance, as implicitly assumed in this work. This large coupling of the two-star $D$-wave $J^{P}=3 / 2^{-} N^{*}(2080)$ resonance to the $\Lambda^{*} K^{+}$channel will confirm/get support from the QM results of Simon Capstick, and W. Roberts in Ref. [15], as mentioned above. We have taken advantage of the apparent important role played by the resonant contribution to explain the SPring-8 LEPS $\vec{\gamma} p \rightarrow K^{+} \Lambda(1520)$ data, and we have used it to improve our knowledge on some $N^{*}(2080)$ properties. First, keeping the electromagnetic $e f_{1}$ and $e f_{2}$ couplings fixed, we have taken the mass and width of the resonance as adjustable parameters. From our six-parameter fit B, we find $2138 \pm 4 \mathrm{MeV}$ and $168 \pm 10 \mathrm{MeV}$ for the resonance mass and width, respectively (we do not quote here the errors induced by the helicity amplitude uncertainties), and a significant

${ }^{5}$ We take $M_{\Lambda^{*}}=1.5195 \mathrm{GeV}$ and $m_{K}=0.4937 \mathrm{GeV}$. 
TABLE I: Values of some of the parameters used/determined in this work. First we give the input used in each of the three fits considered in this work: The electromagnetic couplings $e f_{1}$ and $e f_{2}$ deduced from the $N^{*}$ helicity amplitudes reported in the PDG (the numerical values of the helicity amplitudes are also compiled here) and the mass and the total width of the $N^{*}$ resonance. Next, we give results from different best fits to the $\gamma p \rightarrow K^{+} \Lambda^{*}$ differential cross section data at forward angles and below $E_{\gamma}=2.4 \mathrm{GeV}$ of Ref. [4]. Finally, we also give $\Gamma_{N^{*} \rightarrow \Lambda^{*} K}$ predicted from the parameters of each $\chi^{2}-$ fit, and in the case of the eight-parameter fit, we also show the predicted $N^{*}$ helicity amplitudes.

Input Parameters

\begin{tabular}{|c|c|c|c|}
\hline & Fit A & Fit B & Fit C \\
\hline$A_{1 / 2}^{p^{*}}\left[\mathrm{GeV}^{-1 / 2}\right]$ & $-0.020 \pm 0.008$ & $-0.020 \pm 0.008$ & --- \\
\hline$A_{3 / 2}^{p^{*}}\left[\mathrm{GeV}^{-1 / 2}\right]$ & $0.017 \pm 0.011$ & $0.017 \pm 0.011$ & --- \\
\hline$e f_{1}$ & $0.18 \pm 0.07$ & $0.18 \pm 0.07$ & --- \\
\hline$e f_{2}$ & $-0.19 \pm 0.07$ & $-0.19 \pm 0.07$ & --- \\
\hline$M_{N^{*}}[\mathrm{MeV}]$ & 2080 & --- & --- \\
\hline$\Gamma_{N^{*}}[\mathrm{MeV}]$ & 300 & --- & --- \\
\hline
\end{tabular}

Fitted Parameters

\begin{tabular}{|c|c|c|c|}
\hline$g_{1}$ & $5.0 \pm 0.2_{-1.5}^{+2.8}$ & $2.0 \pm 0.1_{-0.5}^{+1.4}$ & $1.4 \pm 0.3$ \\
\hline$g_{2}$ & $-9.7 \pm 2.0_{-5}^{+6}$ & $-3.3 \pm 0.9_{-3.4}^{+1.8}$ & $5.5 \pm 1.8$ \\
\hline$\Lambda_{s}=\Lambda_{t}[\mathrm{MeV}]$ & $613 \pm 2_{-8}^{+5}$ & $613 \pm 2_{-5}^{+1}$ & $604 \pm 2$ \\
\hline$\Lambda_{R}[\mathrm{MeV}]$ & $990 \pm 50_{-20}^{+30}$ & $5.0 \pm 3.9^{a}$ & $909 \pm 55$ \\
\hline$e f_{1}$ & --- & --- & $0.177 \pm 0.023$ \\
\hline$e f_{2}$ & --- & --- & $-0.082 \pm 0.023$ \\
\hline$M_{N^{*}}[\mathrm{MeV}]$ & --- & $2138 \pm 4_{-21}^{+1}$ & $2115 \pm 8$ \\
\hline$\Gamma_{N^{*}}[\mathrm{MeV}]$ & --- & $168 \pm 10_{-15}^{+19}$ & $254 \pm 24$ \\
\hline$\chi^{2} / d o f$ & 2.4 & 1.4 & 1.2 \\
\hline
\end{tabular}

Predicted Parameters

\begin{tabular}{|c|c|c|c|}
\hline$A_{1 / 2}^{p^{*}}\left[\mathrm{GeV}^{-1 / 2}\right]$ & --- & --- & $0.0036 \pm 0.0086$ \\
\hline$A_{3 / 2}^{p^{*}}\left[\mathrm{GeV}^{-1 / 2}\right]$ & --- & --- & $0.058 \pm 0.021$ \\
\hline$\Gamma_{N^{*} \rightarrow \Lambda^{*} K}[\mathrm{MeV}]$ & $110 \pm 10_{-50}^{+160}$ & $43 \pm 5_{-20}^{+61}$ & $19 \pm 7$ \\
\hline$\frac{\Gamma_{N^{*} \rightarrow \Lambda^{*} K}}{\Gamma_{N^{*}}}[\%]$ & $36 \pm 3_{-18}^{+53}$ & $26 \pm 3_{-12}^{+36}$ & $7.5 \pm 2.8$ \\
\hline
\end{tabular}

${ }^{a}$ In GeV.

improvement $^{6}$ of the $\chi^{2} / d o f$, which is now of the order of 1.4. The major evidences quoted in the PDG for the $N^{*}(2080)$ resonance come from analyses performed thirty years ago [27, 28], and that certainly are not inconsistent with values for the mass and width in the range of 2140 $\mathrm{MeV}$ and $170 \mathrm{MeV}$, respectively. We have also explored the possibility of determining the the electromagnetic $e f_{1}$ and $e f_{2}$ couplings of this resonance, and hence we have carried out an eight-parameter fit (C), where these two couplings are also adjusted to data. The $\chi^{2} /$ dof slightly lowers down to 1.2 , being $e f_{1}$ and $e f_{2}$ nearly compatible, within errors, with those deduced form the helicity amplitudes. Note, that these latter ones were also re-

\footnotetext{
${ }^{6}$ Note that for fit B, we find an unrealistic central value of $5 \mathrm{GeV}$ for the cutoff $\Lambda_{R}$, with a large error $(\sim 4 \mathrm{GeV})$, which indicates that the $\chi^{2}$ is rather insensitive to this parameter. Indeed, we get equivalent fits $\left(\chi^{2} /\right.$ dof $\left.=1.4-1.6\right)$ as long as $\Lambda_{R} \geq 1 \mathrm{GeV}$. The rest of parameters change within their respective errors to accommodate the minor modifications induced by the change in the resonance cut off parameter.
}

ported almost 30 years ago [29], and they might be also subject of large uncertainties. Besides, in this fit, the $g_{1}$ coupling turns out to be significantly smaller than that obtained in the first fit, what leads to a much smaller $\Gamma_{N^{*} \rightarrow \Lambda^{*} K} / \Gamma_{N^{*}}$ branching fraction, probably more reasonable than that deduced from the four parameter fit A. We conclude, that this latter fits leads to an overall good description of data and that it can be used to constrain some of the properties of the $N^{*}(2080)$ resonance. Indeed, this can be seen in Fig. 3, where differential cross sections, deduced from the results of the eight-parameter fit $\mathrm{C}$, are shown and compared to data. In this figure, dashed and dotted lines show the contributions from the background and $N^{*}$ resonance terms, respectively, while the solid line displays the full result. For this latter curve we also show the $68 \%$ CL band obtained from the statistical uncertainties of the fitted parameters. We see that the bump structure in the differential cross section at forward $K^{+}$angles is fairly well described $\left(\chi^{2} /\right.$ dof $\left.\sim 1.2\right)$ thanks to a significant contribution from the $N^{*}$. 

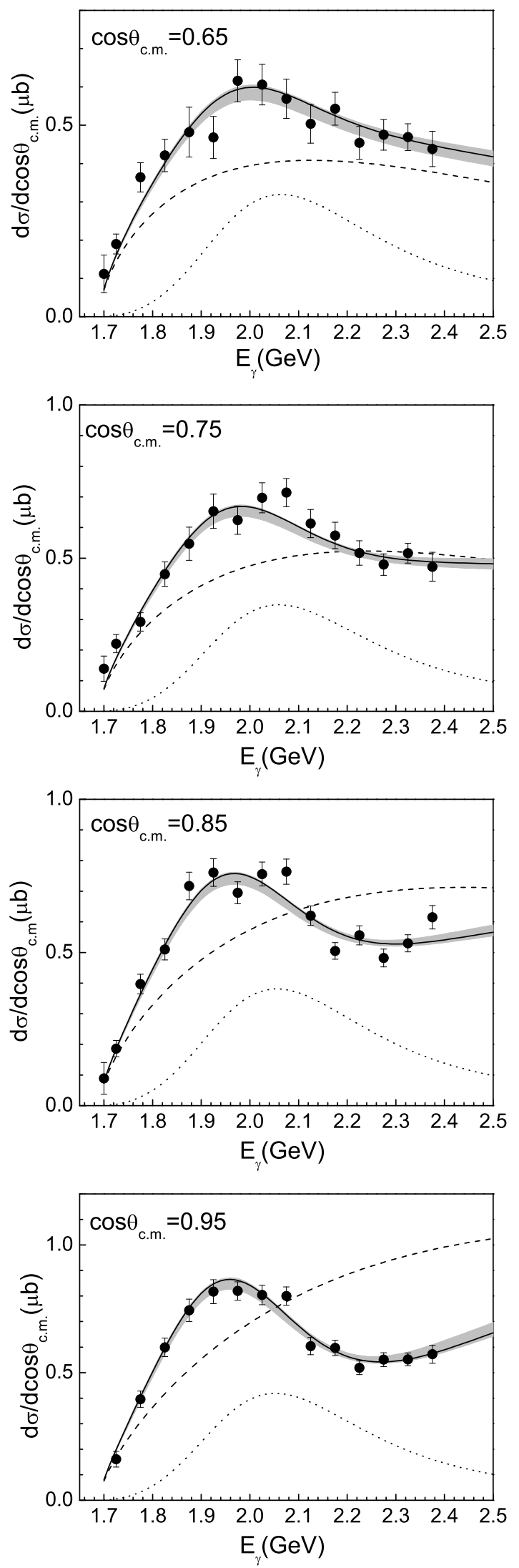

FIG. 3: $\gamma p \rightarrow K^{+} \Lambda^{*}$ differential $d \sigma / d\left(\cos \theta_{\text {C.M. }}\right)$ cross sections compared with the LEPS data [4]. Results have been obtained from the eight-parameter fit $\mathrm{C}$ (details can be found in Table I). Dashed and dotted lines show the contributions from the background and $N^{*}$ resonance terms, respectively, while the solid line displays the full result. For this latter curve we also show the statistical $68 \%$ CL band.
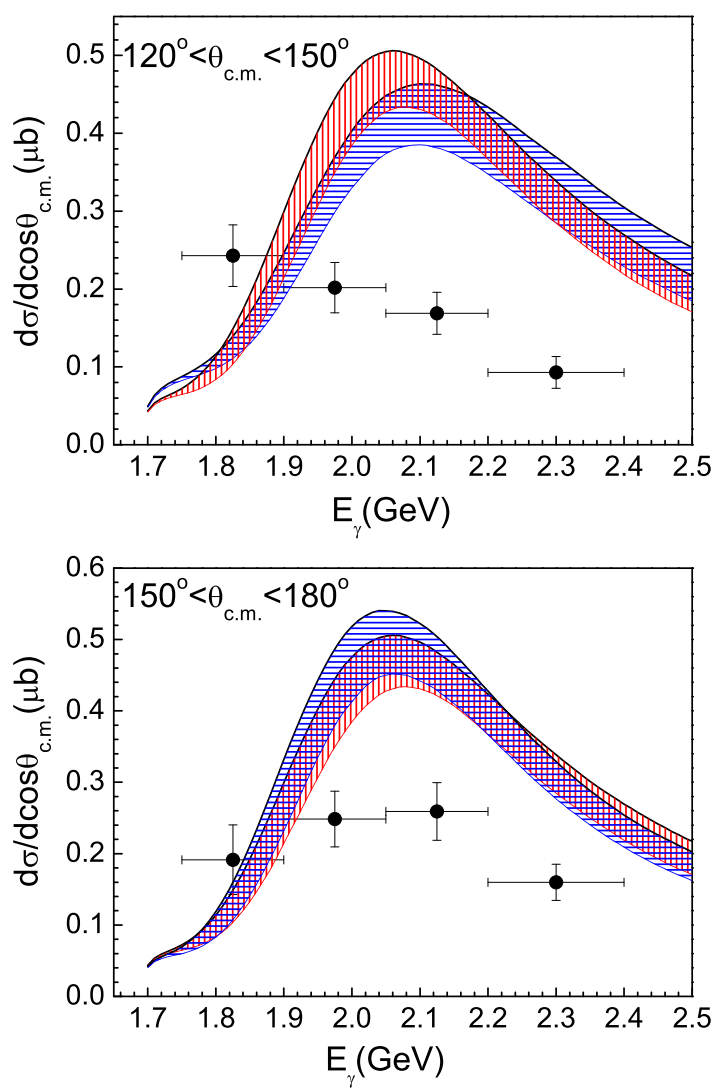

FIG. 4: (Color online) $\gamma p \rightarrow K^{+} \Lambda^{*}$ differential $d \sigma / d\left(\cos \theta_{\text {C.M. }}\right)$ cross section compared with the LEPS data [3]. In both panels, we display our predicted (fit C) $68 \%$ CL bands for the two extreme values of the corresponding $\theta_{\text {C.M. }}$. interval. The blue horizontal line shaded region stand for the $68 \%$ CL band associated to $120^{\circ}$, in the upper panel and to $180^{\circ}$ in the lower panel. In both panels, the red vertical line shaded region stand for the $68 \%$ CL band that corresponds to $150^{\circ}$.

Next, we pay attention to backward angles and in Fig. (4 we depict differential cross sections for large kaon scattering angles and obtained with the fitted (eight) parameters given in the Table [1, as a function of $E_{\gamma}$ and the kaon scattering angle $\theta_{\mathrm{C} . \mathrm{M}}$. The experimental datapoints are taken from Ref. [3], where events were accumulated for two angular intervals $\theta_{\text {C.M. }}=(120-150)^{0}$ and $\theta_{\text {C.M. }}=(150-180)^{0}$, with the photon energy varying in the region $1.9 \leq E_{\gamma} \leq 2.4 \mathrm{GeV}$. In both panels, we have computed our predictions for the two extreme values of the corresponding $\theta_{\mathrm{C} . \mathrm{M}}$. interval. The shaded regions accounts for the uncertainties inherited from those affecting the parameters compiled in Table【. The $68 \%$ CL error bands have been obtained using a MC simulation and they turn out to be bigger than at forward angles because the backward differential cross section is largely dominated by the $N^{*}(2080)$ pole contribution. The full polar angular dependence of the theoretical differential 
cross section is compared with the data of Ref. [3] in Fig. 5] To increase the statistics, data have been integrated over the photon energy interval $1.9 \leq E_{\gamma} \leq 2.4$ $\mathrm{GeV}$. We have considered photons of 1.9 and $2.4 \mathrm{GeV}$ of energy and computed the $68 \%$ CL error bands for each energy. Both in Fig. 4 and 5, we see that our theoretical model leads to reasonable descriptions of the data.

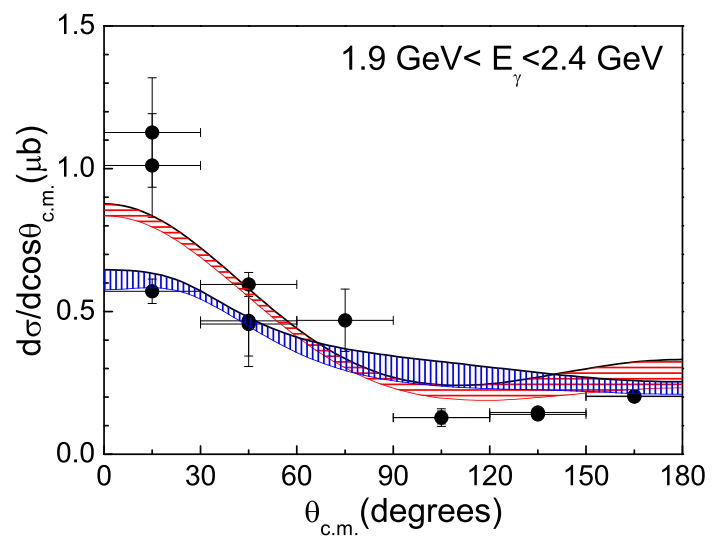

FIG. 5: (Color online) $\gamma p \rightarrow K^{+} \Lambda^{*}$ differential $d \sigma / d\left(\cos \theta_{\text {C.M. }}\right)$ cross section compared with the LEPS data [3]. Shaded regions stand for our predicted (fit C) $68 \%$ CL bands for 1.9 (red horizontal lines) and 2.4 (blue vertical lines) GeV energy photons.

Finally, in Fig. 6, we compare our predictions (fit C) for the polar angle average photon-beam asymmetry $\langle\Sigma\rangle$ as a function of $E_{\gamma}$ with the recent SPring-8 LEPS data of Ref. [4]. We calculate $\langle\Sigma\rangle$ as

$$
\langle\Sigma\rangle=\frac{\int_{0.6}^{1.0} \frac{d \sigma}{d\left(\cos \theta_{\text {C.M. })}\right.} \Sigma\left(\cos \theta_{\text {C.M. }}, E_{\gamma}\right) d\left(\cos \theta_{\text {C.M. }}\right)}{\int_{0.6}^{1.0} \frac{d \sigma}{d\left(\cos \theta_{\text {C.M. }}\right)} d\left(\cos \theta_{\text {C.M. }}\right)},
$$

where $\Sigma\left(\cos \theta_{\text {C.M. }}, E_{\gamma}\right)$ is defined in Eq. (17). In this figure, dashed and dotted lines show the polar angle average asymmetry from the background and $N^{*}$ resonance terms alone, respectively, while the solid line displays the full result. For this latter curve we also show the $68 \%$ CL grey band obtained from the statistical errors of the fitted parameters in Table \. The description of our model of this observable is much poorer than in the rest of cases examined above, and we find here discrepancies of about two standard deviations with data when the theoretical uncertainties are also taken into account. One might think that the inclusion of a $t$-channel $K^{*}$ exchange might improve the situation, since it leads to positive values for the asymmetry [12]. We have explored such a possibility $^{7}$, but we have found tiny changes even for values of the

\footnotetext{
7 We have used Eq. (15) of Ref. [9].
}

$K^{*} \Lambda^{*} N$ coupling constant as large as 10, strongly disfavored by the theoretical findings of Ref. [9], and certainly the inclusion of this mechanism would not significantly improve the situation. We have also studied the effect of including a relative complex phase between the background and the $N^{*}$ contributions. With values of this phase around $130-140^{\circ}$ and re-adjusting the rest of the parameters, we find values of $\langle\Sigma\rangle$, though still negative, much smaller (in absolute values) and closer to zero than those displayed in Fig. 6. Moreover, the fair agreement with the $d \sigma / d \cos \theta_{\text {C.M. }}$. data exhibited in Fig. 3 is not spoiled out, at all.

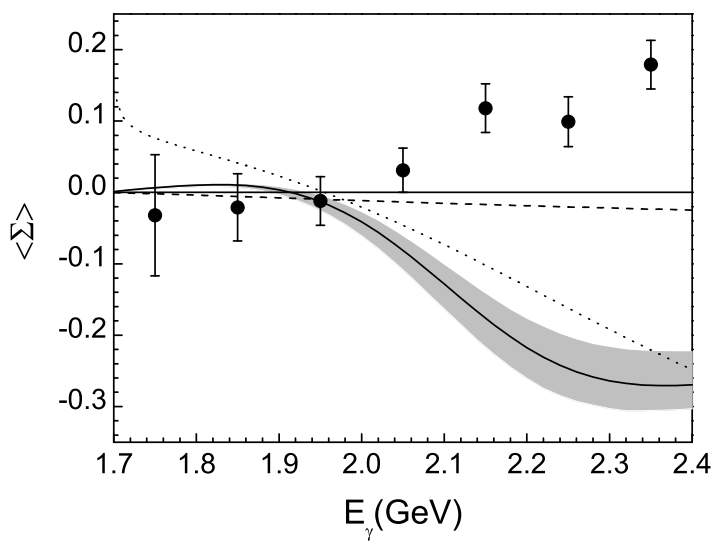

FIG. 6: Polar angle $\left(0.6<\cos \theta_{\text {C.M. }}<1.0\right)$ average photonbeam asymmetry $\langle\Sigma\rangle$ as a function of $E_{\gamma}$ for the $\gamma p \rightarrow K^{+} \Lambda^{*}$ reaction. Dashed and dotted lines show the average asymmetry from the background and $N^{*}$ resonance terms alone, respectively, while the solid line displays the full result (eightparameter fit C). For this latter curve we also show the $68 \%$ CL grey band obtained from the statistical errors of the fitted parameters in Table [ Data have been taken from Ref [4].

We would like to point out that if the sign of our calculated asymmetry is changed, we would find a better description of data. However, in the best of our knowledge our definition of the asymmetry is consistent with that stated in Ref. [4], and given in terms of the ratio $\left(N_{v}-N_{h}\right) /\left(N_{v}+N_{h}\right)$, where $N_{v}$ and $N_{h}$ are the $\Lambda^{*}$ yields with vertically and horizontally polarized photons, respectively. Yet, we would like to mention that the authors of Ref. [16] also find negative values for photon beam asymmetry, as can be seen in Fig. 9a of that reference.

\section{SUMMARY AND CONCLUSIONS}

We have studied the $\vec{\gamma} p \rightarrow \Lambda^{*} K^{+}$reaction at low energies within a effective Lagrangian approach. In particular, we have paid an special attention to a bump structure in the differential cross section at forward $K^{+}$angles 
reported in the recent SPring-8 LEPS experiment [4]. Starting from the background contributions studied in previous works, we have shown that this bump might be described thanks to the inclusion of the nucleon resonance $N^{*}(2080)$ (spin-parity $J^{P}=3 / 2^{-}$). We have fitted its mass, width and hadronic $\Lambda^{*} K^{+}$and electromagnetic $N^{*} N \gamma$ couplings to data. We have found that this resonance would have a large decay width into $\Lambda^{*} K$, which will be compatible with the findings of the QM approach of Ref. 15]. We have also calculated differential cross sections at backward angles and the polar angle average photon-beam asymmetry. In the first case, our results compare reasonably well with data, while for the case of the photon-beam asymmetry the agreement with the experimental measurements is much poorer, and we find discrepancies of about two standard deviations for photon energies above $2 \mathrm{GeV}$. The proposed scheme here, should be supplemented with some other reaction mechanisms which could improve the achieved description of the photon-beam asymmetry data.

Other explanations of the observed bump in the SPring-8 LEPS data are also possible. Indeed, in the very same experimental paper (Ref. [4]) where the data is published, it is suggested that this structure might be due to a $J^{P}=\frac{3}{2}^{+}$nucleon resonance, with a mass of $2.11 \mathrm{GeV}$ and a width of $140 \mathrm{MeV}$. However, a nucleon resonance with these features is not listed in the PDG book [13]. In Ref. [4], it is also mentioned the possibility of a sizeable contribution from a higher $\left(J^{P}=\frac{5}{2}^{-}\right)$baryon state and/or the existence of a new reaction process, for instance, an interference with $\phi$ photo-production 30,31 .

However, we have shown here that the photo- production of the $N^{*}(2080)$ resonance off the proton and its subsequent decay into $\Lambda^{*} K^{+}$might also provide a simple explanation of the bump structure observed in the experimental data. This contradicts the findings of Refs. [8, 16], where unnecessarily small $N^{*}(2080) \Lambda^{*} K^{+}$ couplings and probably a too large width for this resonance were used.

Finally, we would like to stress that thanks to the important role played by the resonant contribution in the $\vec{\gamma} p \rightarrow K^{+} \Lambda(1520)$ reaction, accurate data for this reaction can be used to improve our knowledge on some $N^{*}(2080)$ properties, which are at present poorly known. This work constitutes a first step in this direction.

\section{Acknowledgments}

We warmly thank M.J. Vicente-Vacas and J. Martin Camalich for useful discussions. This work is partly supported by DGI and FEDER funds, under contract FIS2008-01143/FIS, the Spanish Ingenio-Consolider 2010 Program CPAN (CSD2007-00042), and Generalitat Valenciana under contract PROMETEO/2009/0090. We acknowledge the support of the European CommunityResearch Infrastructure Integrating Activity "Study of Strongly Interacting Matter" (acronym HadronPhysics2, Grant Agreement n. 227431) under the Seventh Framework Programme of EU. Work supported in part by DFG (SFB/TR 16, "Subnuclear Structure of Matter"). JuJun Xie acknowledges Ministerio de Educación Grant SAB2009-0116.
[1] D. P. Barber et al. (LAMP2 Collaboration), Z. Phys. C 7, 17 (1980).

[2] A. M. Boyarski et al., Phys. Lett. B 34, 547 (1971).

[3] N. Muramatsu et al. (LEPS Collaboration), Phys. Rev. Lett. 103, 012001 (2009).

[4] H. Kohri et al. (LEPS Collaboration), Phys. Rev. Lett. 104, 172001 (2010).

[5] A. I. Titov, B. Kämpfer, S. Datá, and Y. Ohashi, Phys. Rev. C 72, 035206 (2005); ibid C 74, 055206 (2006).

[6] A. Sibirtsev, J. Haidenbauer, S. Krewald, U.-G. Meißner, and A. W. Thomas, Eur. Phys. J. A 31, 221 (2007).

[7] S. I. Nam, A. Hosaka, and H.-C. Kim, Phys. Lett. B 579, 43 (2004); Phys. Rev. D 71, 114012 (2005): Phys. Lett. B 633, 483 (2006); J. Korean Phys. Soc. 49, 1928 (2006).

[8] S. I. Nam, Phys. Rev. C 81, 015201 (2010).

[9] H. Toki, C. García-Recio, and J. Nieves, Phys. Rev. D 77, 034001 (2008).

[10] C. García-Recio, J. Nieves and L. L. Salcedo, Phys. Rev. D 74034025 (2006).

[11] S. P. Barrow et al. (CLAS Collaboration), Phys. Rev. C 64044601 (2001).

[12] S. I. Nam, K. S. Choi, A. Hosaka and H. C. Kim, Phys. Rev. D 75, 014027 (2007).

[13] C. Amsler et al. Phys. Lett. B 667, 1 (2008).
[14] S. Capstick, Phys. Rev. D 46, 2864 (1992) .

[15] S. Capstick, and W. Roberts, Phys. Rev. D 58, 074011 (1998).

[16] S. I. Nam and C. W. Kao, Phys. Rev. C 81, 055206 (2010).

[17] W. Rarita and J. Schwinger, Phys. Rev. 60, 61 (1941).

[18] L. M. Nath, B. Etemadi, and J. D. Kimel, Phys. Rev. D 3, 2153 (1971).

[19] T. Mizutani, C. Fayard, G. H. Lamot and B. Saghai, Phys. Rev. C 58, 75 (1998).

[20] V. Pascalutsa and R. Timmermans, Phys. Rev. C 60, 042201 (1999).

[21] V. Pascalutsa, Phys. Lett. B 503, 85 (2001).

[22] Yongseok Oh, Che Ming Ko, and K. Nakayama, Phys. Rev. C 77, 045204 (2008).

[23] K. Ohta, Phys. Rev. C 40 (1989) 1335.

[24] H. Haberzettl, C. Bennhold, T. Mart, T. Feuster, Phys. Rev. C 58, R40 (1998).

[25] R. M. Davidson and R. Workman, Phys. Rev. C 63, 025210 (2001).

[26] S. Janssen, J. Ryckebusch, D. Debruyne and T. Van Cauteren, Phys. Rev. C 65, 015201 (2002).

[27] G. Hohler, F. Kaiser, R. Koch and E. Pietarinen, "Handbook Of Pion Nucleon Scattering", 
http://www.slac.stanford.edu/spires/find/hep/www?irn=644803, to 10th Int. Symp. on Lepton and Photon Interactions at

[28] R. E. Cutkosky, C. P. Forsyth, J. B. Babcock, R. L. Kelly and R. E. Hendrick, "Pion - Nucleon Partial Wave Analysis", presented at 4th Int. Conf. on Baryon Resonances, Toronto, Canada, Jul 14-16, 1980.

[29] N. Awaji et al., "Energy Dependent Partial Wave Analysis On Single Pion Photoproduction", contributed paper High Energy, Bonn, West Germany, Aug 24-28, 1981.

[30] S. Ozaki, A. Hosaka, H. Nagahiro, and O. Scholten, Phys. Rev. C 80, 035201 (2009).

[31] A. Kiswandhi, Ju-Jun Xie, and S. N. Yang, Phys. Lett. B 691, 214 (2010). 